\title{
Gut acellular matrix for the in vitro study of Enteric Nervous System cells
}

\author{
Schrenk $\mathrm{S}^{1}$, Piccione $\mathrm{M}^{1}$, Tasso $\mathrm{A}^{1}$, Di Liddo $\mathrm{R}^{1,2, *}$ and Conconi $\mathrm{MT}^{1,2}$ \\ ${ }^{1}$ Department of Pharmaceutical and Pharmacological Sciences, University of Padova, 35131, Padova (PD), Italy \\ ${ }^{2}$ Foundation for Biology and Regenerative Medicine, Tissue Engineering and Signaling (TES) ONLUS, 35030, Padova, Italy \\ \#The authors contributed equally
}

\begin{abstract}
Enteric nervous system (ENS) cells respond to the intestinal extracellular matrix (ECM) signals changing their proliferation rate, migration and differentiation. In this study, we explored in vitro the adaptive response of primary ENS cell cultures to the stimulation of gut acellular matrix (AM) defining the gene expression profile of neuronal functionality markers. Scanning electron microscopy was used to detect the acquisition of specific morphological features.

Intestinal AM was prepared using an enzyme-detergent treatment. Primary rat enteric cells were isolated from the myenteric plexus of postnatal rats using an enzymatic method and seeded on intestinal AM in the presence of exogenous neurotrophic factors. The morphological properties and the expression of specific differentiation markers were evaluated by Scanning Electron Microscopy (SEM) and wholemount fluorescent staining. In order to verify the synergic activity of soluble factors and AM, the gene expression of neurotransmitter receptors was evaluated by qPCR in ENS cells cultured in SM conditions in the presence or not of AM.

The development of interconnected ganglion-like structures and the expression of neurotransmitter receptors suggested that gut matrix engineered with ENS cells could be useful for medical applications of regenerative medicine or for the in vitro assessment of tridimensional culture system of ENS.
\end{abstract}

\begin{abstract}
Abbreviations: ENS: Enteric Nervous System; ECM: Extracellular Matrix; AC: Acellular Matrix; SEM: Scanning Electron Microscopy; qPCR: quantitative Polymerase Chain Reaction; ENSc: Enteric Nervous System cells; IBD: Inflammatory Bowel Disease; DAPI: 4',6-diamidino-2-phenylindole; IF: Immunofluorescence; H\&E: haematoxylin/eosin; Avprla: Arginine vasopressin receptor 1A; Gabra1: Gamma-aminobutyric acid (GABA) A receptor, alpha 1; Gabre: Gamma-aminobutyric acid (GABA) A receptor, epsilon; Grin2a: Glutamate receptor, ionotropic, N-methyl D-aspartate 2A; Htr2a: 5-hydroxytryptamine (serotonin) receptor 2A; Sstr1: Somatostatin receptor 1; Sstr2: Somatostatin receptor 2; Tspo: Translocator protein; Actb: Actin, beta; Ldha: Lactate dehydrogenase A; Rplp: Ribosomal protein, large, P1; Adra2a: Adrenergic, alpha-2A-, receptor; Avpr1b: Arginine vasopressin receptor 1B; Cckbr: Cholecystokinin B receptor; Chrm1: Cholinergic receptor, muscarinic 1; Chrna3: Cholinergic receptor, nicotinic, alpha 3; Drd2: Dopamine receptor D2; Drd5: Dopamine receptor D5; Gabra5: Gamma-aminobutyric acid (GABA) A receptor, alpha 5; Gabra6: Gamma-aminobutyric acid (GABA) A receptor, alpha 6; Grin2c: Glutamate receptor, ionotropic, N-methyl D-aspartate 2C; Grm4: Glutamate receptor, metabotropic 4; Hrh4: Histamine receptor H4; Htrla: 5-hydroxytryptamine (serotonin) receptor 1A; Htr1b: 5-hydroxytryptamine (serotonin) receptor 1B; Npy5r: Neuropeptide Y receptor Y5; Ntsr2: Neurotensin receptor 2; Sstr4: Somatostatin receptor 4; Tacr1: Tachykinin receptor 1; B2m: Beta2 microglobulin; Brs3: Bombesin-like receptor 3; Chrne: Cholinergic receptor, nicotinic, epsilon; Cnr1: Cannabinoid receptor 1 (brain); Gabbr1: Gamma-aminobutyric acid (GABA) B receptor 1; Gabbr2: Gamma-aminobutyric acid (GABA) B receptor 2; Gabra2: Gammaaminobutyric acid (GABA) A receptor, alpha 2; Gria3: Glutamate receptor, ionotropic, AMPA 3; Grpr: Gastrin releasing peptide receptor;
\end{abstract}

Hcrtr2: Hypocretin (orexin) receptor 2; Htr7: 5-hydroxytryptamine (serotonin) receptor 7; Tacr3: Tachykinin receptor 1; NCCs: Neural Crest Cells; GDNF: Glial cell-Derived Neurotrophic Factor; NGF: Nerve Growth Factor; bFGF: basic Fibroblast Growth Factor; EGF: Epidermal Growth Factor; NMDA: N-methyl-D-aspartate; AMPA: a-amino3-hydroxyl-5-methyl-4-isoxazole-propionate; PBS: Phosphate Salt Buffer; DNase-I: deoxyribonuclease-I; BSA: Bovine Serum Albumin; RT: Retro-Transcription; FITC: Fluorescein Isothiocyanate; CGM: Complete Growth Medium.

\section{Introduction}

In the last decade, the acellular matrices obtained by a detergentenzymatic method [1], have been demonstrated to optimize in vitro and in vivo cell viability providing a near-physiologic environment. Showing high structural heterogeneity for responding to mechanical, chemical, nutritional, immunological and bacterial stimuli, ECM serves to organize cells in microenvironment, to direct site-specific cellular regulation and to elicit cellular responses, such as proliferation and differentiation. Rather than merely providing structural information, it plays an instructive role that is critical for the maintenance of tissue

*Correspondence to: Rosa Di Liddo, Department of Pharmaceutical and Pharmacological Sciences, University of Padova, Via Marzolo 5, I-35131, Padova, Italy, E-mail: rosa.diliddo@unipd.it

Key words: ENS, extracellular matrix, gut acellular matrix

Received: November 04, 2019; Accepted: November 22, 2019; Published: November 25, 2019 
homeostasis or the initiation and progression of pathologies. To date, the influence exerted by ECM in the regulation of Enteric Nervous System is far from being clear. Under inflammatory bowel diseases (IBD) [1-5], dysfunctional motility, hypersensitivity and interruptions in the neuronal network are commonly observed and correlated to ECM disruption [6-8]. In this context, the development of a threedimensional cellular model could be beneficial to investigate ENSgut matrix interactions under in vivo-mimicking in vitro conditions. In the past decade, ENS progenitor cells isolated from rodents or humans [9-13] have been successively used to assess two-dimensional neurospheres cultures. Lacking an adequate scaffold, ENS cells are considered as limited to study in vitro the mechanisms underlining gut homeostasis or diseases.

Biological matrices obtained by decellularization of native tissues [14] have been successfully used in pre-clinical animal studies or clinical applications of regenerative medicine [14-17]. Under in vitro settings, AMs support cell adhesion, growth, differentiation and functionality $[18,19]$ due to a network architecture resembling native ECM [20]. After implantation, compelling evidence has shown their ability to target tissue specific regeneration [17,21]. In this work, we adapted the decellularization method defined by Conconi et al. (2005) to obtain an optimized rat 3D culture system based on gut acellular matrix and primary ENS cells [22]. Providing cells with intestinal adequate architecture, matrix composition and exogenous soluble factors, this model could be useful for researchers to gain more translatable and clinically relevant data in the research of gut dysfunctions.

\section{Materials and methods}

All animal procedures were performed according to D.Lgs $n$. 26/2014 which warrants care of experimental animals in Italy. The research project was approved by the Italian Health Department according to the art. 26 and 31 of above-mentioned D.Lgs.

\section{Gut acellular matrix}

Intestines were obtained from adult Sprague Dawley rats (3-months-old) and rinsed four times in phosphate-buffered saline (PBS), 1\% antifungal and antibiotic agent (Amuchina, sodium hypochlorite, Angelini ACRAF S.p.A., Ancona, Italy). Specimens from small intestine were treated according to Conconi et al. [19]. Briefly, the luminal surface of each sample was treated with $4 \mathrm{U} / \mathrm{mL}$ Dispase II (Roche Diagnostics, Monza, Italy) to remove the epithelial monolayer. Then, six decellularization cycles were performed as follows: sterile Milli-Q water supplemented with $1 \%$ of antibiotic solution (SigmaAldrich, Milan, Italy) for $72 \mathrm{~h}$ at $4^{\circ} \mathrm{C}, 4 \%$ sodium deoxycholate (Sigma-Aldrich) for $4 \mathrm{~h}$, and $2000 \mathrm{kU}$ deoxyribonuclease-I (DNase-I) (Sigma-Aldrich) in $1 \mathrm{M} \mathrm{NaCl}$ (Sigma-Aldrich) for $3 \mathrm{~h}$. Matrices were stored in $\mathrm{PBS}$ at $4^{\circ} \mathrm{C}$ until use. The presence of cellular elements was verified histologically [haematoxylin/eosin (H\&E) (Sigma-Aldrich) and DAPI staining (Invitrogen, Thermo Fisher Scientific, Monza, Italy)] on cryosections. In parallel, the presence of DNA and RNA was investigated digesting tissue specimens with TRIzol Reagent (Invitrogen), according to the manufacturer's instructions. The analysis was performed using a NanoDrop 2000 spectrophotometer (Thermo Fisher Scientific) and DNA/RNA samples extracted from native intestines were used as reference. Acellular matrices (AMs) were stored at $4^{\circ} \mathrm{C}$, in PBS supplemented with $1 \%$ antibiotic solution.

\section{Haematoxylin/Eosin}

AMs were longitudinally cut to obtain samples of approximately 5 $\mathrm{mm}^{2}$. After fixation in cryostat embedding medium (Killik, Bio-Optica,
Milano, Italy), sections with $7 \mu \mathrm{m}$ thickness were prepared using a Cryostat DM2000 (Leica Microsystems) and transferred on glass microscopic slides. Staining with haematoxylin/eosin (H\&E, SigmaAldrich) was carried out according to standard protocols.

\section{Scanning electron microscopy (SEM)}

For the morphological study, AMs were fixed with $4 \%$ glutaraldehyde (Merck, Darmstadt, Germany) in $0.1 \mathrm{M}$ cacodylate buffer (pH 7.2) (Prolabo, Paris, France) for $24 \mathrm{~h}$. After dehydration in ethanol, the samples were submitted to critical point drying and gold sputtering for the examination by a scanning electron microscope (Stereoscan-205 S, Cambridge Instruments, Cambridge, UK).

\section{Immunofluorescence (IF)}

Seven-micron thick sections were permeabilized with Triton X-100 (Sigma-Aldrich) for $30 \mathrm{~min}$. To prevent non-specific binding of antibodies, blocking was performed by incubation in PBS supplemented with $1 \%(\mathrm{w} / \mathrm{v})$ bovine serum albumin (BSA, Sigma-Aldrich). Then, the samples were incubated with rabbit anti-rat laminin antibody (CosmoBio, Tokyo, Japan) overnight, at room temperature (RT). After washing in PBS, binding specific sites were revealed by incubation with secondary antibody goat anti-rabbit-FITC (Santa Cruz Biotechnologies, Inc., Heidelberg, Germany) at $4^{\circ} \mathrm{C}$, for $1 \mathrm{~h}$. Negative controls were prepared treating AM sections with only secondary antibody. After mounting with Anti-Fade Mounting Medium (Immunological Sciences, Rome, Italy), the analysis was performed using a DM2000 (Leica Microsystems, Wetzlar, Germany).

\section{Isolation and culture of ENS cells (ENSc)}

ENSc were isolated from intestine of 3-day-old Sprague Dawley rats according to Schäfer et al. [23]. Overlying tissue was stripped out and the smooth muscle layer was extracted for digestion at $37^{\circ} \mathrm{C}$ in Hank's balanced salt solution (PAN, Aidenbach, Germany), $50 \mathrm{ng} / \mathrm{ml}$ trypsinchymotrypsin inhibitor (Sigma-Aldrich), $1 \mathrm{mg} / \mathrm{mL}$ collagenase type 2 (Worthington, Lakewood, USA) and $200 \mu \mathrm{g} / \mathrm{mL}$ DNAse (Roche, Basel, Switzerland). Myenteric nets were collected and then treated with $1 \mathrm{X}$ Accutase (PAA, Pasching, Austria) at $37^{\circ} \mathrm{C}$, for $10 \mathrm{~min}$. The cells were seeded $\left(2 \times 10^{5}\right.$ cells $\left./ \mathrm{cm}^{2}\right)$ and cultured for seven days under standard conditions in complete growth medium (CGM) composed of Neuronal Base P (PAA) 2\% (v/v) neuronal stem cell supplement (PAA), 1\% (v/v) bovine serum albumin (Sigma-Aldrich), $0.1 \%$ (v/v) $\beta$-mercaptoethanol (Invitrogen), 1\% (v/v) penicillin/streptomycin (Invitrogen) and 1\% (v/v) L-glutamine (Sigma-Aldrich), $10 \mathrm{ng} / \mathrm{mL}$ EGF (ImmunoTools, Friesoythe, Germany), $20 \mathrm{ng} / \mathrm{mL}$ b-FGF (ImmunoTools) and $10 \mathrm{ng} / \mathrm{mL}$ GDNF (ImmunoTools).

\section{Assessment of 3D model cell culture}

AMs were cut into pieces of approximately $5 \mathrm{~mm}^{2}$, stretched on a glass coverslip and then transferred into 24 -well plates (Falcon, BD Biosciences, Milan, Italy). Stainless steel rings were used to anchor the samples to the bottom of plates. Primary neurospheres were obtained by culturing ENS cells for 7 days and, after enzymatic disruption with 1X Accumax solution (Sigma-Aldrich) for $15 \mathrm{~min}$ at $37^{\circ} \mathrm{C}$, were mechanically dissociated using a 27 Gauge needle. Single cell suspensions were seeded at a density of $2 \times 10^{4}$ cells $/ \mathrm{cm}^{2}$ on the outer side of AMs. At three different time points (3, 7 and 14 days), the three-dimensional cultures were fixed and prepared for SEM analysis, wholemount immunofluorescent staining and gene expression analysis. 


\section{Wholemount immunofluorescence staining}

The samples were fixed using BD Cytofix (BD Biosciences, Milan, Italy) for $15 \mathrm{~min}$ and $1 \%(\mathrm{v} / \mathrm{v}) \mathrm{BSA}$ for $2 \mathrm{~h}$ at $4^{\circ} \mathrm{C}$ for blocking unspecific binding sites. For intracellular staining, the incubation with $0.5 \%(\mathrm{v} / \mathrm{v})$ Triton X-100 (Sigma-Aldrich) was performed for 30 min, at RT. The incubation with mouse anti-rat tubulin $ß$ III (Merck Millipore, Vimodrone (MI), Italy) and rabbit anti-rat $\alpha$-SMA-FITC (Abcam, Cambridge, UK) antibodies was executed overnight, at RT. The detection of tubulin $B$ III required the incubation for $3 \mathrm{~h}$ at RT with a secondary antibody goat anti-mouse PE (Santa Cruz Biotechnology). After mounting with Anti-Fade Mounting Medium (Immunological Sciences), the samples were examined using a fluorescence microscope DM2000 (Leica Microsystems, Wetzlar, Germany).

\section{Gene expression study}

Total RNA was extracted using RNeasy Mini Kit (Qiagen, Milan, Italy) and quantified with a NanoDrop 2000 spectrophotometer (Thermo Fisher Scientific). Gene expression analysis was performed using $\mathrm{RT}^{2}$ Profiler ${ }^{\mathrm{m}}$ PCR Array Rat Neurotransmitter Receptors (Qiagen, Hilden, Germany), according to manufacturer's instructions. The list of target genes is reported in Supplementary data, Table S1). RNA isolated from ENSc cultured on polystyrene plates was used as control. The Rat neurotransmitter receptor $\mathrm{RT}^{2}$ Profiler PCR array profiles the expression of 84 important cytokine genes. Data were acquired using DNA Engine Opticon ${ }^{\star}$ Real Time Thermal Cycler (MJ Research, St. Bruno, QC, Canada) and analyzed using the $\mathrm{RT}^{2}$ Profiler data analysis Web portal (SABiosciences, Washington DC, US), applying the $2^{-\Delta \Delta \mathrm{Ct}}$ method. Hypoxanthine phosphoribosyl transferase 1 (Hprt1) was used as housekeeping gene.

\section{Statistical analysis}

The $p$-values were calculated based on a Student's t-test of the replicate applied on $2^{-\Delta \Delta \mathrm{Ct}}$ values for each gene in the control group and treatment group. Fold-change values less than one have been considered a down-regulation; fold-change values greater than two have been interpreted as an up-regulation. Data were analyzed using the $\mathrm{RT}^{2}$ Profiler data analysis Web portal (SABiosciences, Washington DC, US), applying the $2^{-\Delta \Delta \mathrm{Ct}}$ method. In the analysis, $\mathrm{p}<0.05$ was considered statistically significant.

\section{Results}

\section{Gut matrices}

In comparison to the native gut (Figure 1A), samples submitted to six cycles of decellularization showed to be lacking mucosal/ submucosal layers (Figure 1B). Compared to controls (Figure 1C), no signs of nuclei and cytoplasmic components were detected in AMs by DAPI staining (Figure 1D). Furthermore, more than 99\% of nucleic acid content (DNA: $2.82 \pm 0.4 \mathrm{ng} / \mu \mathrm{L}$; RNA: $13.17 \pm 4.99 \mathrm{ng} / \mu \mathrm{L}$ ) was removed by decellularization with respect to the native gut (DNA: $523.20 \pm 11.31 \mathrm{ng} / \mu \mathrm{L}$; RNA: $1552.04 \pm 15.23 \mathrm{ng} / \mu \mathrm{L}$ ) (see graph in Supplementary data). By scanning electron microscopy, the outermost layer showed a flat surface, with fibers longitudinally arranged (Figure $1 \mathrm{E}$ ) and gut architecture almost preserved (Figure 1F), as suggested by the detection of laminin through Immunofluorescence (IF) (see Supplementary data).

\section{SEM}

After 3 days of culture, ENSc were attached and spread on AMs (Figure 2A). From 7 (Figure 2B) to 14 days (Figure 2C), numerous colonies started to acquire morphological specialization and ganglionlike interconnected structures were detectable.

\section{Immunofluorescence (IF)}

In parallel, wholemount immunofluorescent staining confirmed the ganglion-like appearance and the positivity for the expression of tubulin $\beta$ III, a typical structural component of neuronal connection and differentiation during 3 (Figure 3A), 7 (Figure 3B) and 14 (Figure $3 C)$ days. The presence of cells positive for the expression of $\alpha$-Smooth Muscle Actin ( $\alpha$-SMA) and negative for tubulin $\beta$ III underlined the ability of ENSc to differentiate also into glial-smooth muscle like cells.

\section{Gene expression profile}

PCR data confirmed that AMs compared to the traditional bidimensional culture system enhance the in vitro functionality of ENSc. The analysis performed with $\mathrm{RT}^{2}$ Profiler PCR Array (Figure 4) showed a significant expression change (fold change $>2$ with $p$-value $<0.05$ ) in 29 of 84 total analyzed genes in ENSc cultured on AMs compared to control samples on polystyrene dishes. The expression resulted increased for 11 genes (Avpr1a, Gabra1, Gabre, Grin2a, Htr2a, Sstr1, Sstr2, Tspo, Actb, Ldha and Rplp), while a decreased expression was detected for 18 targets (Adra2a, Avpr1b, Cckbr, Chrm1, Chrna3, Drd2, drd5, Gabra5, Gabra6, Grin2c, Grm4, Hrh4, Htr1a, Htr1b, Npy5r, Ntsr2, Sstr4 and Tacr1). Twelve genes resulted to be unaffected by the culture

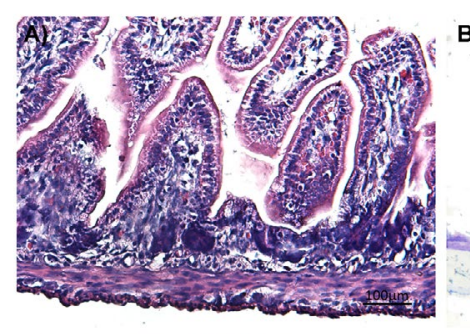

B)
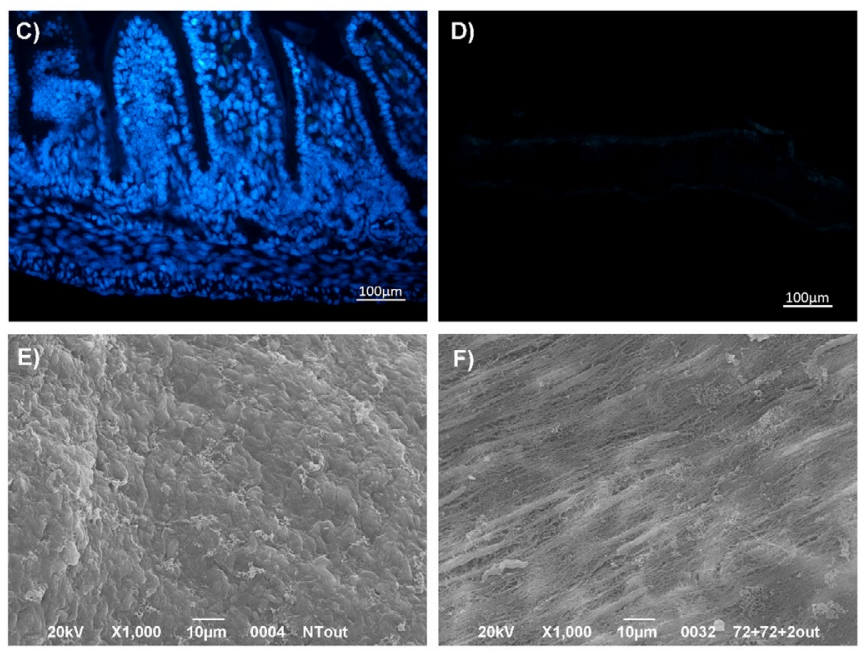

Figure 1. The decellularization protocol removed $\sim 99 \%$ of the cellular content from rat gut. Morphological study by H\&E staining of native gut (A) and intestinal acellular matrix (B). DAPI staining of intestine (C) and gut acellular scaffolds (D). SEM analysis of gut external layer before (E) and after decellularization procedure (F). Magnification: $\mathrm{x} 100$ 
system (B2m, Brs3, Chrne, Cnr1, Gabbr1, Gabbr2, Gabra2, Gria3, Grpr, Hcrtr2, Htr7, Tacr3) while remaining 47 targets were not expressed or expressed at non-detectable level (Ct-value $>35$ ) in both culture conditions. Data from this analysis were reported in a Volcano plot showing fold-change and $p$-value for ENSc seeded on AMs compared to cultures on polystyrene dishes. Upregulated and downregulated genes were indicated with red and blue dots, respectively. Genes with unchanged expression level were reported with black dots.
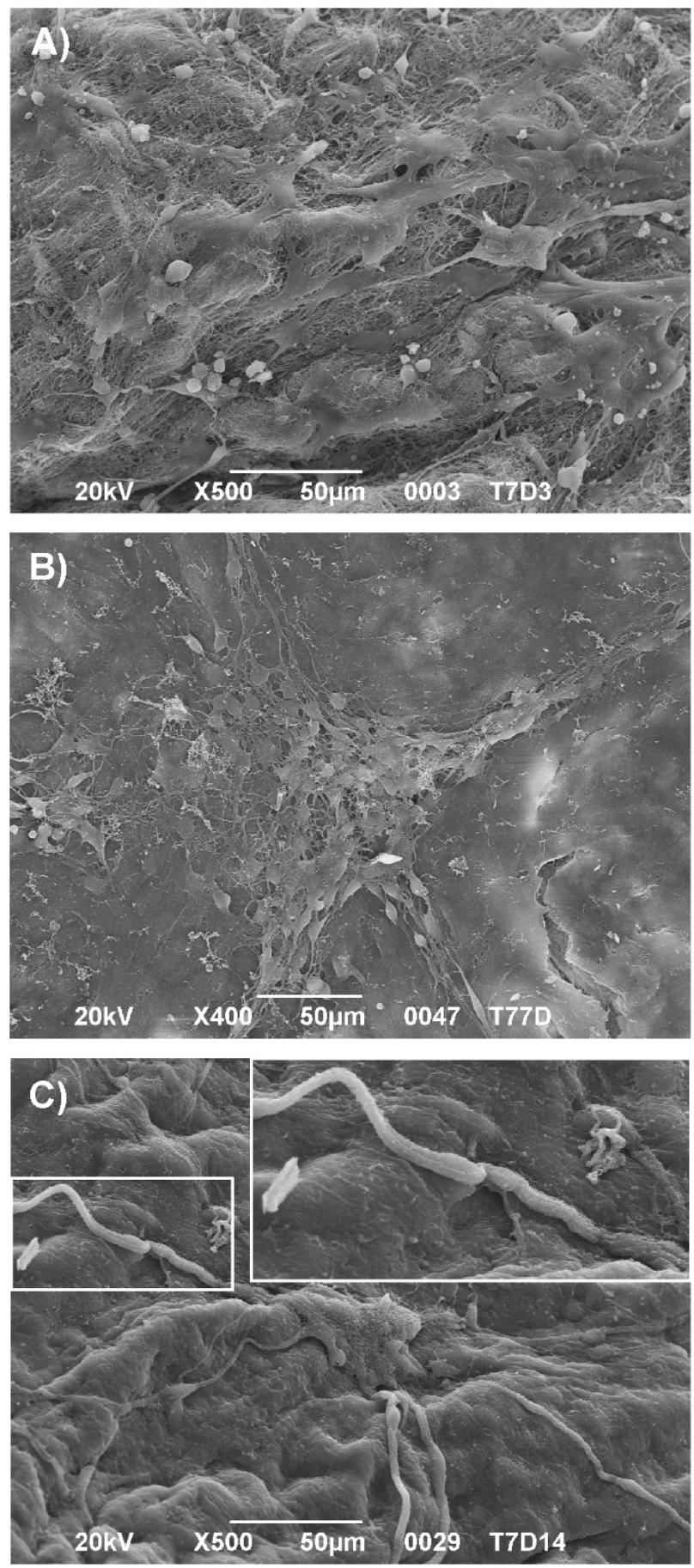

Figure 2. ENS cells formed ganglion-like structures on acellular matrices. SEM analysis at 3 (A), 7 (B) and 14 (C) days of culture on AM. Magnification: X100
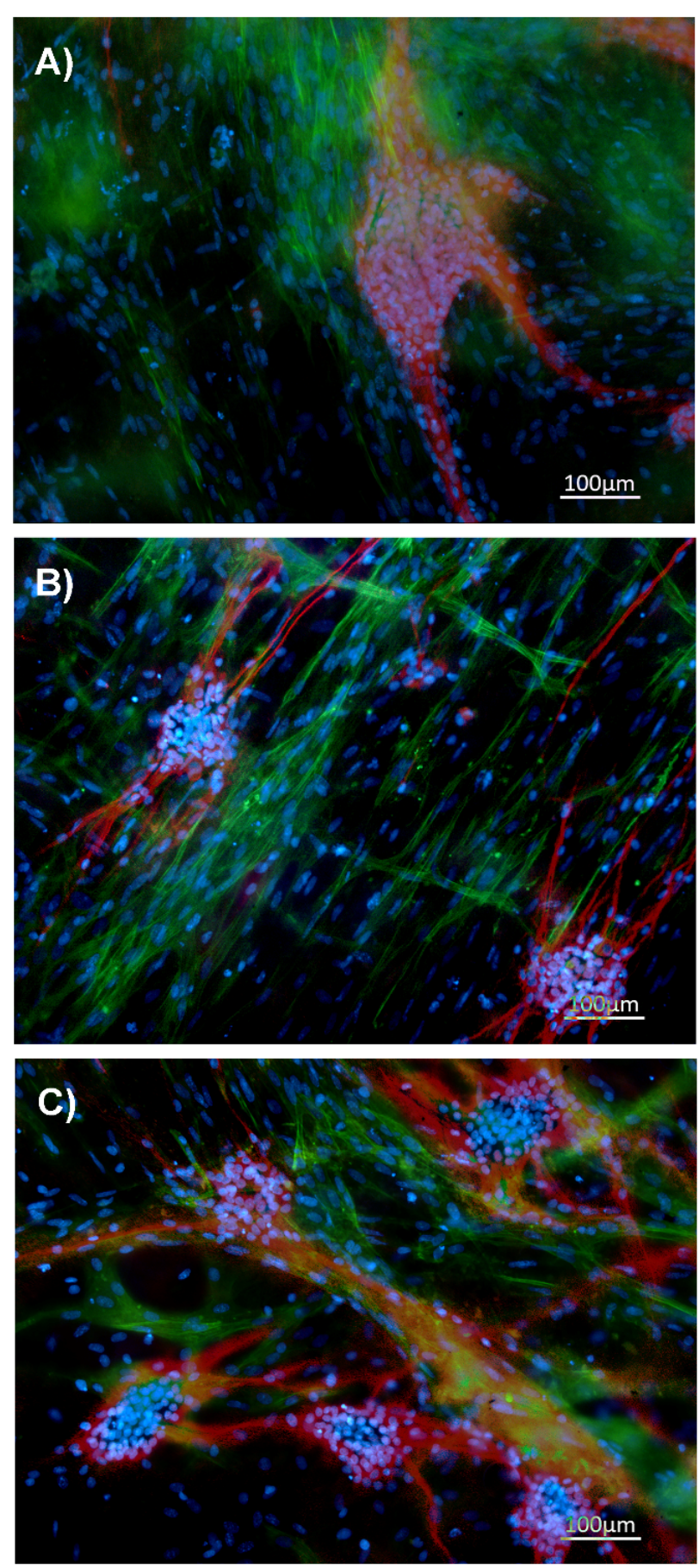

Figure 3. ENS cultured on acellular matrix expressed tubulin $\beta$ III. Immunofluorescence analysis of tubulin $\beta$ III (red), $\alpha$-SMA (green), DAPI (blue) on ENS cells cultured for 3 (A), 7 (B) and 14 (C) days on acellular matrices. Magnification: x100

\section{Discussion}

Biologic scaffolds obtained from tissue or organ decellularization have been successfully used in both pre-clinical studies and human clinical applications [24]. In the last decade, several decellularization protocols have been investigated to define the optimal method for obtaining acellular matrices with minimal residual cellular material and low-grade ultrastructure alterations [14]. In this paper, gut ECM 


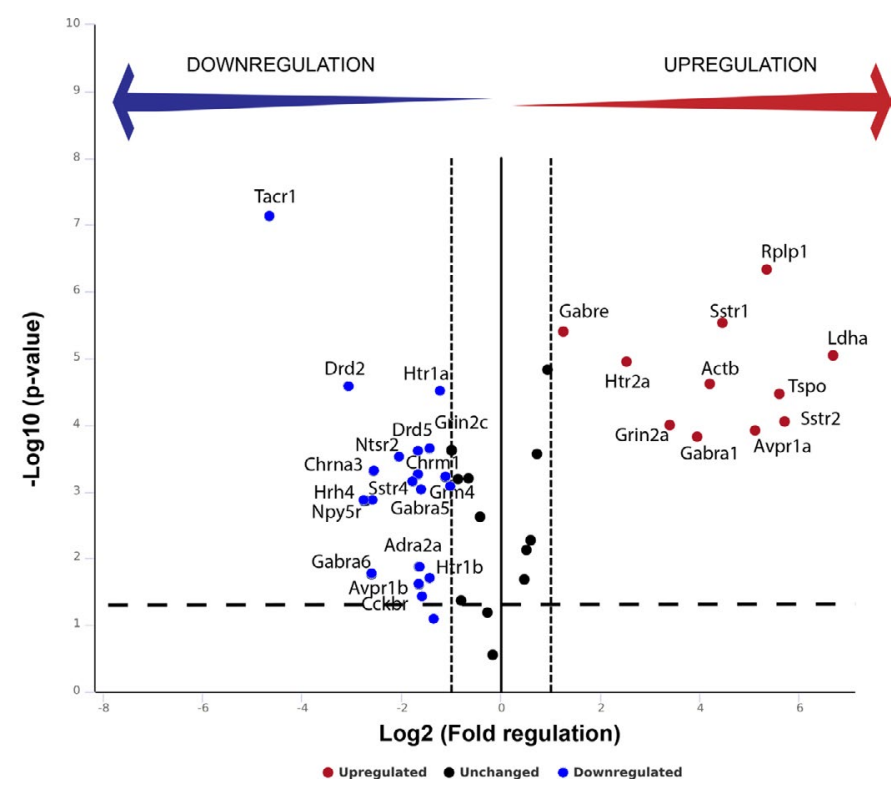

Figure 4. Genes related to neuronal signaling are differently expressed in ENS cells cultured on AMs or polystyrene. The expression of 84 neurotransmitter receptors genes was analyzed by a RT ${ }^{2}$ Profiler PCR array. RT-qPCR data were analyzed using the DDCt method with $\mathrm{Ct}$ value $<35$. Each dot in volcano plot is representative of one target gene: the $\mathrm{x}$-axis indicates the biological impact (fold change in ENSc-AM culture system compared to controls on polystyrene), whereas the y-axis reports the statistical reliability of the fold change (the $p$-value). The upregulated genes were represented in red; while the downregulated ones were reported in blue. Genes that were unaffected by the culture systems were shown in black. Data were representative of three independent experiments

scaffolds were prepared from rat intestine according to Conconi et al. (2005) and then used for culturing in vitro ENS-derived cells [19]. The supplementation of culture medium with growth factors was also considered to further improve the cell functionality and differentiation potential. The development of ENS is a highly dynamic process involving the migration of neural crest cells (NCCs) in developing gut and the extensive proliferation/differentiation of neuronal and glial progenitors [25]. It is known that a small subset of NCCs persists in ENS of postnatal or adult gut, serving as a backup system that continuously supplies the intestine with specialized cells. Compelling evidence has demonstrated that soluble factors such as Glial cellDerived Neurotrophic Factor (GDNF) [24,25], Nerve Growth Factor (NGF) [26-28], basic Fibroblast Growth Factor (bFGF) [29,30], Epidermal Growth Factor (EGF) are essential for NCCs growth and also to optimize in vitro the survival [9-11,31] or differentiation [3234] of ENS progenitor cells of rodents or humans. Furthermore, gut ECM, that is mainly composed of collagen IV, fibronectin, laminin and heparan-sulphate proteoglycans [35], has long been known to provide the necessary ultrastructural cues for the formation of enteric ganglia [34-36] and neurotrophic signaling [37-40]. In accordance to Raghavan et al. [41], that defined the impact of ECM components on neuronal and glial differentiation, the preserved laminin in AMs confirmed the potentialities of the biologic scaffolds to sustain the outgrowth, the differentiation and the survival of neurons or progenitors [13,40-43]. Transcriptome study of ENS cultured on AMs compared to control cells established differences in the expression profile of genes responsible for GABA (Gabre, Gabra1) Glutamate (Grin2a) and Serotonin (Htr2a). In parallel, the significant increase of Somatostatin signaling through Sstr1 and Sstr2 was also observed providing the evidence that ECM components synergistically act with bioactive soluble factors to mimic native intestine microenvironment and drive the commitment of immature cells to different subtypes of neurons [44]. Although through different receptors, control cells showed to be active in the development of neuron-like cells with high responsivity to Dopamine, Serotonin and GABA but not to glutamate, confirming the potentialities of AM to enhance neuronal in vitro differentiation. A remarkable finding of the present study was that some genes, such as L-lactate dehydrogenase A chain (Ldha), that is notably related to energetic metabolism and, converting pyruvate to lactate during aerobic glycolysis. Yang et al. reported that L-lactate stimulates in neurons the expression of genes related to synaptic plasticity through a mechanism involving the activation of N-methyl-D-aspartate (NMDA) receptors, such as Grin2a [45]. Moreover, it also protects neurons against Glutamate-mediated excitotoxicity [46]. Acellular matrix was demonstrated to positively modulate the gene expression of beta-Actin $(A c t b)$, a protein recently reported to contribute to neural crest ontogeny [47], directional growth cone motility [46-50] and development of dendritic spine processes [51]. The active process of neuronal maturation was strongly confirmed by the formation of tubulin $\beta$ III-positive ganglion-like structures on acellular matrix.

\section{Conclusions}

The synergic activity exerted by intestinal AM and neurotrophic factors promoted the maturation of ENS cells into interconnected ganglion-like structures expressing genes related to Acetylcholine, GABA, Glutamate, Serotonin and Somatostatin receptors. Our findings validate an effective approach of cell/matrix-based system to potentiate in vitro the differentiation abilities of ENS cells.

\section{Author contributions}

Study conception and design, C.M.T.; Execution of experiments, S.S., P.M., and T.A.; Collection and assembly of data: S.S. and P.M.; Manuscript writing, S.S., P.M., and D.L.R; Manuscript editing: P.M., T.A., D.L.R., and C.M.T.; Data analysis: C.M.T.; D.L.R.; Data interpretation and final approval of manuscript: D.L.R. and C.T.M.

\section{Acknowledgments}

All the authors acknowledge a special thanks to Dr. Bertalot Thomas for the technical collaboration.

\section{Conflicts of interests}

Authors declare no conflicts of interest.

\section{References}

1. Meezan E, Hjelle JT, Brendel K, Carlson EC (1975) A simple, versatile, nondisruptive method for the isolation of morphologically and chemically pure basement membranes from several tissues. Life Sci 17: 1721-1732.

2. Vasina V (2006) Enteric neuroplasticity evoked by inflammation. Auton Neurosci 126127: $264-272$

3. Mawe GM, Strong DS, Sharkey KA (2009) Plasticity of enteric nerve functions in the inflamed and postinflamed gut. Neurogastroenterol Motil 21: 481-491.

4. Lakhan SE, Kirchgessner A (2010) Neuroinflammation in inflammatory bowel disease. J Neuroinflammation 7: 37. [Crossref]

5. Villanacci V (2008) Enteric nervous system abnormalities in inflammatory bowel diseases. Neurogastroenterol Motil 20: 1009-1016.

6. Geboes K and Collins S (1998) Structural abnormalities of the nervous system in Crohn's disease and ulcerative colitis. Neurogastroenterol Motil 10: 189-202.

7. Isgar B, Harman M, Kaye MD, Whorwell PJ (1983) Symptoms of irritable bowel syndrome in ulcerative colitis in remission. Gut 24: 190-192.

8. Gracie DJ, Ford AC (2017) Ongoing symptoms in ulcerative colitis patients in remission: irritable bowel syndrome or gastrointestinal symptoms in the absence of inflammation? Inflamm Bowel Dis 23: E4-E5. 
9. Beyak MJ and Vanner S (2005) Inflammation-induced hyperexcitability of nociceptive gastrointestinal DRG neurones? the role of voltage-gated ion channels. Neurogastroenterol Motil 17: 175-186.

10. Rauch U, Hansgen A, Hagl C, Holland-Cunz S, Schafer KH (2006) Isolation and cultivation of neuronal precursor cells from the developing human enteric nervous system as a tool for cell therapy in dysganglionosis. Int J Colorectal Dis 21: 554559.

11. Grundmann D, Klotz M, Rabe H, Glanemann M, Schafer KH (2015) Isolation of highpurity myenteric plexus from adult human and mouse gastrointestinal tract. Sci Rep 5: 9226 .

12. Schafer KH (1997) A new method for the isolation of myenteric plexus from the newborn rat gastrointestinal tract. Brain Res Protoc 1: 109-113.

13. Heanue TA and Pachnis V (2011) Prospective identification and isolation of enteric nervous system progenitors using Sox2. Stem Cells 29: 128-140.

14. Crapo PM (2011) An overview of tissue and whole organ decellularization processes. Biomaterials 32: 3233-3243.

15. Chen LV (2018) Decellularized bone matrix scaffold for bone regeneration. Methods Mol Biol 1577: 239-254. [Crossref]

16. Giuffrida P (2019) Decellularized Human Gut as a Natural 3D platform for research in intestinal fibrosis. Inflamm Bowel Dis 2: 1-11.

17. Matoug-Elwerfelli M, Duggal MS (2018) A biocompatible decellularized pulp scaffold for regenerative endodontics. Int Endod J 51: 663-673.

18. Dall'Olmo L (2014) Blood Vessel-derived acellular matrix for vascular graft application. Biomed Res Int.

19. Conconi MT (2005) Tracheal matrices, obtained by a detergent-enzymatic method, support in vitro the adhesion of chondrocytes and tracheal epithelial cells Transpl Int 18: 727-734.

20. De Carlo E (2010) Pancreatic acellular matrix supports islet survival and function in a synthetic tubular device? In vitro and in vivo studies Int J Mol Med 25: 195-202.

21. Rana D, Zreiqat H, Benkirane-Jessel N (2017) Development of decellularized scaffolds for stem cell-driven tissue engineering. J Tissue Eng Regen Med 11: 942-965.

22. Conconi MT (2008) In vitro and in vivo evaluation of acellular diaphragmatic matrices seeded with muscle precursors cells and coated with VEGF silica gels to repair muscle defect of the diaphragm. J Biomed Mater Res.

23. Schafer KH (1997) A new method for the isolation of myenteric plexus from the newborn rat gastrointestinal tract Brain Res Protoc 1: 109-113.

24. Macchiarini P (2008) Clinical transplantation of a tissue-engineered airway. Lancet 372: $2023-2030$.

25. Lake JI and Heuckeroth RO (2013) Enteric nervous system development: Migration, differentiation, and disease. Liver Physiol 305: 359-362.

26. Moore MW, Klein RD, Farinas I, Sauer H, Armanini M, et al. (1996) Renal and neuronal abnormalities in mice lacking GDNF. Nature 382: 76-79. [Crossref]

27. Schafer KH (1999) The GDNF-induced neurite outgrowth and neuronal survival in dissociated myenteric plexus cultures of the rat small intestine decreases postnatally. Exp Brain Res 125: 447-452.

28. Mulholland MW (1994) Nerve growth factor promotes neurite outgrowth in guinea pig myenteric plexus ganglia. Liver Physiol 267: 30-34.

29. Lourenssen S (2005) Selective loss of NGF-sensitive neurons following experimental colitis. Exp Neurol 191: 337-343.

30. Kuroda T (1994) Altered Production of Nerve Growth Factor in Aganglionic Intestines J Pediatr Surg 29: 288-293.
31. Chadi G (2004) Basic fibroblast growth factor, neurofilament, and glial fibrillary acidic protein immunoreactivities in the myenteric plexus of the rat esophagus and colon. $J$ Morphol 333: 323-333.

32. Hagl CI (2013) FGF2 deficit during development leads to specific neuronal cell loss in the enteric nervous system Histochem Cell Biol 139: 47-57.

33. Heanue TA (2011) Prospective identification and isolation of enteric nervous system progenitors using Sox2. Stem Cells 29: 128-140.

34. Sato Y (2008) Retinoic acid regulates murine enteric nervous system precursor proliferation, enhances neuronal precursor differentiation, and reduces neurite growth in vitro. Dev Biol 320: 185-198.

35. Bannerman PGC (1988) Antigenic markers and laminin expression in cultured enteric neural cells. Brain Res 440: 87-98

36. Pomeranz HD (1993) Neural crest-derived cells isolated from the gut by immunoselection develop neuronal and glial phenotypes when cultured on laminin. Developmental Biology 156: 341-361.

37. Tennyson VM (1997) A 1 subunit of Laminin-1 promotes the development of neurons by interacting with LBP110 expressed by neural crest-derived cells immunoselected from the fetal mouse gut. $J$ Neurobiol 33: 118-138.

38. Letourneau PC (1994) Interactions of developing neurons with the extracellular matrix. J Neurosci 14: 915-928.

39. Simon-Assmann P (1995) Extracellular matrix components in intestinal development. Experientia 51: 883-900.

40. Adams JC, Watt FM (1993) Regulation of development and differentiation by the extracellular matrix. Development 117: 1183-1198. [Crossref]

41. Raghavan S (2014) The influence of extracellular matrix composition on the differentiation of neuronal subtypes in tissue engineered innervated intestinal smooth muscle sheets. Biomaterials 31: 7429-7440.

42. Luckenbill-Edds L (1997) Laminin and the mechanism of neuronal outgrowth. Brain Res Brain Res Rev 23: 1-27. [Crossref]

43. Baptista PM (2009) Whole Organ Decellularization - A Tool for Bioscaffold Fabrication and Organ Bioengineering. IEEE 6526-6529.

44. Hao MM (2009) Development of enteric neuron diversity. J Cell Mol Med 13: 1193 1210.

45. Yang J, Ruchti E, Petit JM, Jourdain P, Grenningloh G, et al. (2014) Lactate promotes plasticity gene expression by potentiating NMDA signaling in neurons. Proc Natl Acad Sci U S A 111: 12228-12233. [Crossref]

46. Jourdain P (2016) L-Lactate protects neurons against excitotoxicity: Implication of an ATP-mediated signaling cascade. Sci Rep 6: 1-13.

47. Tondeleir D, Noelanders R, Bakkali K, Ampe C (2014) Beta-actin is required for proper mouse neural crest ontogeny. PLoS One 9: e85608. [Crossref]

48. Yao J (2006) An essential role for $\beta$-actin mRNA localization and translation in $\mathrm{Ca} 2+$ dependent growth cone guidance. Nat Neurosci 9: 1265-1273.

49. Shestakova EA (2001) The physiological significance of ß-actin mRNA localization in determining cell polarity and directional motility. Proc Natl Acad Sci USA 98: 7045 7050 .

50. Kislauskis EH (1994) Sequences responsible for intracellular localization of B-actin messenger RNA also affect cell phenotype. J Cell Biol 127: 441-451.

51. Buxbaum R (2014) Single B-Actin mRNA detection in neurons reveals a mechanism for regulating its translatability. Science 80: 419-422.

Copyright: (C2019 Schrenk S. This is an open-access article distributed under the terms of the Creative Commons Attribution License, which permits unrestricted use, distribution, and reproduction in any medium, provided the original author and source are credited. 